REVISTA DE DERECHO UNED, NÚM. 22, 2018

\title{
REPERCUSIÓN DE LA DIRECTIVA 2016/2341 EN LOS FONDOS DE PENSIONES DE EMPLEO
}

\author{
IMPACT OF DIRECTIVE 2016/2341 \\ ON EMPLOYMENT PENSION FUNDS
}

\author{
MANUELA LaBORDA IBÁÑEZ \\ Doctorando investigadora. \\ Programa de Doctorado en Derecho y Ciencias Sociales. \\ Universidad Nacional de Educación a Distancia (UNED)
}

Resumen: A lo largo de este artículo vamos a analizar la repercusión normativa en el ordenamiento jurídico español de la Directiva 2016/2341, en cuanto que afecta a los fondos de pensiones de empleo en materia de supervisión, gobernanza e información a participes y beneficiarios. Prestaremos especial atención a todo lo que afecta al órgano de control de los fondos de pensiones de empleo, denominado comisión de control. El funcionamiento de esta comisión de control es básico para el sistema de planes y fondos de pensiones de empleo, ya que, este órgano de gobierno es el que supervisa el cumplimiento de las cláusulas del plan y del fondo de pensiones.

Throughout this article we are going to analyze the normative repercussion in the Spanish legal system of the Directive 2016/2341, insofar as it affects the pension funds of employment in the matter of supervision, governance and information to participants and beneficiaries. We will pay special attention to everything that affects the supervisory body of the employment pension funds, which is the control commission. The operation of the control commission is basic to the system of pension plans and funds of employment, since this governing body is the one that supervises compliance with the clauses of the plan and the pension fund. 
Palabras clave castellano: fondos, pensiones, empleo, gobernanza y comisión de control.

Palabras clave inglés: fund, pension, employment, governance and control commission.

Recepción original: 14/11/2017

Aceptación original: 21/02/2018

Sumario: I. Introducción. II. Sistema de gobernanza. II.A. Funciones clave. II.A.1. Gestión de riesgos. II.A.2. Auditoria interna. II.A.3. Función actuarial. II.B. Capacitación. II.C. Autoevaluación. II.D. Depositaria. III. Información a partícipes y beneficiarios. IV. Supervisión prudencial. V. Conclusiones

\section{INTRODUCCIÓN}

El primer paso legislativo de la Unión Europea (UE) relacionado con los fondos de pensiones fue la Directiva 2033/41/CE (IORP I), que supuso un cambio hacia un mercado interior de la previsión ocupacional para la jubilación organizada a escala de la UE. La Directiva que ahora analizamos trata de reforzar ese mercado centrándose en algunas consideraciones básicas como la mejora de la gobernanza y de la gestión del riesgo, así como la revisión de las actividades transfronterizas. Podremos ir viendo a lo largo de este artículo, las modificaciones que nos trasladan desde la UE para que adaptemos nuestra legislación al entorno europeo.

El segundo paso ha sido la publicación de la Directiva 2016/2341, relativa a las actividades y supervisión de los fondos de pensiones de empleo, también conocida por IORP II (Institutions for Occupational Retirement Provision Directive), con la finalidad de conseguir una armonización mínima en materia de Fondos de Pensiones de Empleo (FPE).

Los Fondos de Pensiones son un patrimonio que se crea al objeto de dar cumplimiento a los planes de pensiones de empleo, que son los que su promotor es la empresa y sus partícipes son los empleados de ésta.

El objetivo de esta Directiva es que los FPE operen en distintos Estados miembros, garantizando un elevado nivel de protección y seguridad a los partícipes y beneficiarios de los planes de pensiones. Esta norma espera garantizar la buena gobernanza, la transparencia y la seguridad de los planes de pensiones, aspirando a trasladar a los fondos de pensiones de empleo el nuevo enfoque de gerencia/gestión de riesgos contenido en la Directiva Solvencia II, aplicable a las enti- 
dades de seguros, estableciendo lógicamente las adaptaciones necesarias y guardando la debida proporción con el tamaño, la naturaleza, la escala y la complejidad de las actividades de los FPE.

La norma europea, aspira a garantizar la transparencia y seguridad de los fondos de pensiones de empleo desarrollando tres aspectos básicos:

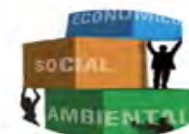

\section{SISTEMA DE GOBERNANZA}

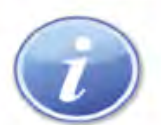

\section{INFORMACIÓN A PARTÍCIPES Y BENEFICIARIOS}

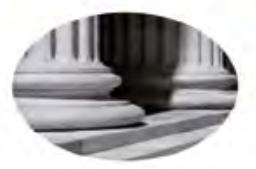

\section{SUPERVISIÓN PRUDENCIAL}

\section{SISTEMA DE GOBERNANZA}

La Directiva, además de regular importantes elementos de la gestión de los fondos de pensiones, introduce como novedad fundamental la elevación a norma con rango de Ley, de los sistemas de Gobernanza. Así pues, se consolida la integración de los criterios no financieros (ya existentes en nuestra regulación, en el artículo 69.5 del Real Decreto 304/2004, por el que se aprueba el Reglamento de Planes y Fondos de Pensiones, RPFP) en los fondos de pensiones más allá de la mera obligación de transparencia o cumplimiento administrativo. Esta Directiva establece que el sistema de gobernanza, incluirá la «ponderación» de los factores ambientales, sociales y de gobernanza relacionados con los activos en las decisiones de inversión, y estará sujeto a una revisión interna periódica.

De conformidad con la Directiva, los Estados miembros deberán exigir a los FPE que indiquen explícitamente si esos factores, (ambientales, sociales y de gobernanza) se toman en consideración en las decisiones sobre inversión y la manera en que forman parte de su sistema de gestión del riesgo. Esta información incluirá la relevancia y el nivel de importancia de los factores citados anteriormente. 
En esta línea, a principios de 2016, se planteó una consulta a la Dirección General de Seguros y Fondos de Pensiones (DGSFP), desde el Foro Español de Inversión Socialmente Responsable (Spainsif), que es una asociación creada con el objetivo de desarrollar y fomentar la Inversión Socialmente Responsable (ISR), que detectó que, en nuestro actual marco legislativo, la regulación sobre la información de los riesgos extrafinancieros, que se facilita por parte de las entidades gestoras, con carácter general, es dispersa y heterogénea.

Por ello, esta asociación propuso un modelo donde los Fondos de Pensiones, de forma voluntaria, pudieran incluir la información de un modo concreto, que la hiciera más homogénea, ya que, si todos los fondos de pensiones dan la información de esta forma, se conseguirá que los datos sean contrastables y la información sea comprensible de una forma mucho más práctica y fructífera. Este modelo de información, haría que los fondos de pensiones pudieran cumplimentar de forma sencilla la información, con identificaciones estandarizadas; posteriormente, esto podría traducirse en información productiva para el sector en general y para los propios fondos de pensiones en particular.

El objetivo es que este modelo de información lo cumplimenten los fondos de pensiones y lo acompañen a la información estadístico contable que, las gestoras de fondos de pensiones, tienen obligación de presentar durante el primer cuatrimestre del año, por ello, si los FPE siguen esta recomendación, se estarán aproximando mucho a las obligaciones que impone la nueva norma europea.

La respuesta a la consulta realizada al supervisor, se emitió con fecha 24 de mayo de 2016, contestando de manera positiva a la pregunta, en el siguiente sentido:

«Se valora, a su vez, de forma positiva la existencia de unas instituciones que permiten aclarar posibles diferencias interpretativas y lograr homogeneidad en las respuestas.

Actualmente la heterogeneidad, tanto cuantitativa como cualitativa, de las informaciones aportadas por los fondos de pensiones para dar cumplimiento al artículo 69.5 del RPFP hace difícil un análisis estadístico de ésta. El que las entidades incorporen en el informe de gestión la información sobre ISR utilizando unos mismos cuadros, permitiría contar con el mismo tipo de información por parte de todos los fondos de pensiones, referenciada además a los mismos conceptos, lo que claramente facilitaría este análisis.

Por todo ello, se considera que los referidos cuadros constituyen una propuesta muy interesante, si bien no podemos olvidar su carácter vo- 
luntario, al no regularse en la normativa de planes de fondos de pensiones. ${ }^{1}$

Es por ello que, en nuestro entorno jurídico, ya tenemos las bases para una parte de este nuevo sistema de gobernanza al que nos tenemos que adaptar de conformidad con la nueva Directiva IORP II.

\section{II.A. Funciones clave}

La Gobernanza sería el primero de los elementos centrales en los que vamos a dividir este artículo; a grandes rasgos, podemos decir que la médula del sistema de gobernanza, reposa sobre las denominadas por la nueva Directiva IORP II funciones clave. Estas son, de conformidad con la redacción que se da en las definiciones de texto de la Directiva: "función clave": dentro de un sistema de gobernanza, la capacidad para llevar a cabo tareas concretas que incluyan la función de gestión de riesgos, la función de auditoría interna y la función

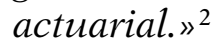

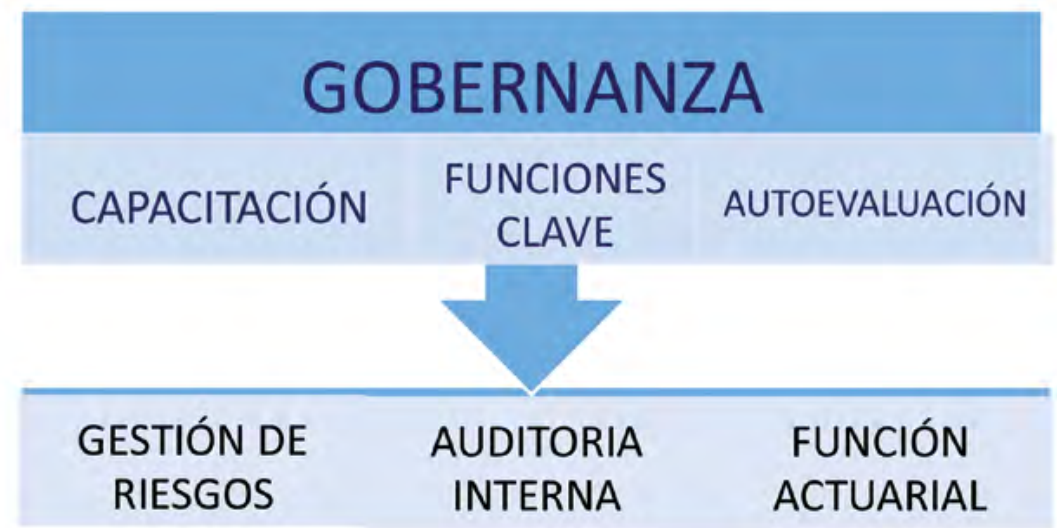

La articulación de estos procedimientos de Gobernanza persigue una gestión integral de los riesgos que afectan en la dinámica de estos patrimonios y que es deseable en todo procedimiento de gestión, pero

${ }^{1}$ Subdirección General de Planes y Fondos de Pensiones. Consulta Número NRF: $1492 / 2016$.

${ }^{2}$ Artículo 6 de la Directiva (UE) 2016/2341 del Parlamento Europeo y del Consejo de 14 de diciembre de 2016 relativa a las actividades y la supervisión de los fondos de pensiones de empleo (FPE). 
debemos tener en cuenta que, en nuestra normativa interna, ya existen controles de gestión de riesgos, y por ello, nuestra adaptación deberá ser menos intensa.

En nuestro caso, los controles de riesgos están profesionalizados, tanto el control de gestión de riesgos que lo asumen las entidades gestoras de fondos de pensiones y que se aplican en la gestión de los fondos, como su revisión que se lleva a cabo en los informes de revisión financiera actuarial con carácter anual o trianual, que realizan empresas o profesionales independientes, y finalmente, en la auditoria de las cuentas anuales, que deben ser emitidas por un auditor independiente.

Es por ello que, a pesar de estar totalmente de acuerdo con el afianzamiento de una función más eficaz en los riesgos asumidos por estos fondos de pensiones, la trasposición que se lleve a cabo de esta Directiva a nuestro ordenamiento jurídico debería ser mínima por la situación de profesionalización en el ámbito de gestión de los riesgos en los fondos de pensiones.

Este sistema de gobernanza se va a centrar en las funciones clave, el artículo 24 de la Directiva, exige a los Estados miembros que requieran a los Fondos de Pensiones de Empleo para que dispongan de las funciones clave que pasamos a detallar:

\section{II.A.1. Gestión de riesgos}

Gestión de riesgos: se incluyen las estrategias, procesos y procedimientos de información para detectar, medir, vigilar, gestionar y notificar de forma continua los riesgos a los que este expuesto el Fondo y deberá incluir al menos los siguientes ámbitos:

— la suscripción y la constitución de reservas;

— la gestión de activos y pasivos;

— la inversión, en particular, en instrumentos derivados, titulizaciones y compromisos similares;

— la gestión del riesgo de liquidez y de concentración;

— la gestión del riesgo operativo;

- el seguro y otras técnicas de reducción del riesgo;

- los riesgos ambientales, sociales y de gobernanza relacionados con la cartera de inversiones y su gestión; 
Como ya hemos dicho, al existir ya controles de gestión de riesgos se reduce el impacto de la Directiva, especialmente en lo que se refiere y pueda afectar al órgano de gobierno de los fondos de pensiones españoles, que es la comisión de control, la cual participa en la elaboración de la Declaración de los principios de la Política de Inversión (DPPI), que es donde se exponen y regulan los riesgos del fondo de pensiones, además de los controles internos que estas entidades tienen.

\section{II.A.2. Auditoria interna}

Auditoría interna: debe evaluar la adecuación y eficacia del sistema de control interno y de otros aspectos del sistema de gobernanza, incluyendo las actividades externalizadas.

Esta segunda función clave, también la tenemos implantada en nuestro sistema de planes y fondos de pensiones y consiste en elaborar las cuentas anules e informe de gestión que tomen en consideración cada uno de los planes que integran el fondo, ofreciendo una imagen fiel de los activos, compromisos y situación financiera del FPE.

\section{II.A.3. Función actuarial}

Función actuarial: que deberá prever una función eficaz llevada a cabo por profesionales independientes, a fin de:

- coordinar y supervisar el cálculo de las provisiones técnicas;

- evaluar la idoneidad de los métodos y modelos subyacentes utilizados para calcular las provisiones técnicas y las hipótesis empleadas al efecto;

- evaluar la suficiencia y la calidad de los datos utilizados en el cálculo de las provisiones técnicas;

- cotejar las hipótesis en que se basa el cálculo de las provisiones técnicas con la experiencia;

- informar al órgano de administración, de dirección o de supervisión del FPE sobre la fiabilidad y la adecuación del cálculo de las provisiones técnicas;

- pronunciarse sobre la política general de suscripción en caso de que el FPE cuente con política de este tipo; 
- pronunciarse sobre la idoneidad del régimen de seguro en caso de que el FPE cuente con un régimen de este tipo;

- contribuir a la aplicación efectiva del sistema de gestión de riesgos;

También esta tercera función la tenemos prevista en nuestra legislación, así pues, habrá únicamente que redefinir su funcionamiento, por lo que no partimos de cero a la hora de llevar a cabo la trasposición de la Directiva.

Nuestra normativa interna prevé una gestión de riesgos realizada por la entidad gestora conjuntamente con la comisión de control; una auditoría que abarcará los aspectos contables y financieros, incluyendo un pronunciamiento expreso en lo relativo al cumplimiento de lo previsto en la regulación de planes y fondos de pensiones que llevarán incorporado un informe anual de gestión del fondo de pensiones de empleo que deberá recoger, entre otras cosas, la política ejercida en relación con los criterios de inversión socialmente responsable; el procedimiento seguido para su implantación, gestión y seguimiento indicando el porcentaje de la cartera del fondo que se invierte en activos que tengan en consideración este tipo de criterios extrafinancieros, por todo esto, con la Directiva únicamente profundizamos en su regulación, pero no nos supone ninguna novedad, tal y como hemos citado anteriormente.

Acabando con las funciones clave, nuestro ordenamiento jurídico, también prevé una revisión financiera actuarial, que tendrá que ser adaptada en su contenido, pero que paradójicamente va más allá de la solicitada por la norma europea, ya que, en el artículo 23 del RPFP se regula la revisión financiero actuarial para todo tipo de planes de empleo, para los de aportación definida y mixtos cada tres años, y para los planes de prestación definida cada año. Decimos que va más allá, porque la Directiva prevé esta función actuarial únicamente para los planes que cubran riesgos biométricos o garanticen el resultado de las inversiones, esto es, para planes de prestación definida o mixtos.

Como órgano de dirección, la comisión de control, de conformidad con nuestro sistema regulador, ya asume ciertas responsabilidades sobre el FPE. Ahora, el reto es estructurarlas de tal forma, que las tres funciones clave queden coordinadas, y se implementen las medidas necesarias para llevar a cabo los aspectos aún no previstos en nuestra normativa. 


\section{II.B. Capacitación}

En relación con la capacitación, otro de los aspectos englobados en el sistema de Gobernanza, nos encontramos con una novedad, la Directiva estable unos requisitos de competencia, idoneidad y honorabilidad para las personas que dirijan de manera efectiva los FPE y para las personas que desarrollen las funciones clave que acabamos de exponer. Nuestra normativa también establece ciertos requisitos en esta materia, aunque la norma comunitaria va más allá, exigiendo una evaluación tanto de los riesgos que afronta la institución como de la capacidad de las personas que los gestionan. Por ello, los miembros de las comisiones de control se verán afectados por esta nueva regulación que les obligará a estar capacitados como personas que dirigen de manera efectiva el FPE. Nuestro sistema de planes y fondos de pensiones exige que la gestión de las inversiones sea realizada por personas honorables que posean la adecuada cualificación y experiencia profesional.

La Directiva, establece para el desempeño de su cometido a las partes integrantes del FPE los siguientes aspectos:

a) requisito de competencia:

i) en el caso de las personas que dirijan de manera efectiva el FPE, sus cualificaciones, competencias y experiencia serán idóneas colectivamente para poder garantizar una gestión adecuada y prudente del FPE,

ii) en el caso de las personas que realicen funciones actuariales o de auditoría interna clave, sus cualificaciones profesionales, sus conocimientos y su experiencia serán idóneos para desempeñar correctamente sus funciones clave,

iii) en el caso de las personas que realicen otras funciones clave, sus cualificaciones, sus conocimientos y su experiencia serán idóneos para desempeñar correctamente sus funciones clave,

b) requisito de honorabilidad: deberán ser personas íntegras y de buena reputación.

Por lo tanto, la parte de los profesionales que gestionan o desarrollan las funciones clave, tal y como hemos visto, ya la tenemos regulada en nuestro ordenamiento jurídico. En cambio, la parte que se refiere a las personas que dirijan de manera efectiva los FPE, refiriéndose en nuestro caso, a los miembros de las comisiones de 
control, no está prevista, y su transposición puede resultar compleja. Por ello, será necesario regular un sistema de capacitación de los miembros del órgano de gobierno de los FPE que se ajuste a nuestro sistema y que sea proporcionado al conjunto de las circunstancias que rodean a los planes y fondos de pensiones en España, ya que, los miembros de las comisiones de control, no son profesionales, sino que son representantes de los trabajadores designados desde la negociación colectiva para desempeñar estas funciones, y no siempre cuentan con los recursos suficientes para llevar a cabo sus tareas.

La Directiva también exigirá a los Estados que establezcan y apliquen una política de remuneración adecuada respecto de todas las personas que dirijan de manera efectiva y de las que desempeñen funciones clave y de otras categorías de personal cuyas actividades profesionales incidan de forma significativa en el perfil de riesgo del Fondo de Pensiones de Empleo. Esta nueva regulación europea puede resultar controvertida en nuestro sistema de planes y fondos de pensiones, que prohíbe expresamente la remuneración de los cargos representativos de las comisiones de control de los fondos de pensiones estableciendo que "El mandato de los miembros de una comisión será temporal y gratuito». ${ }^{3}$

\section{II.C. Autoevaluación}

El penúltimo aspecto de este bloque de gobernanza es la autoevaluación, que consiste en elaborar, al menos cada tres años, un informe de autoevaluación de riesgos, que deberá contener, los siguientes aspectos:

- descripción de cómo se integra la propia valoración de riesgos en el proceso de gestión y en los procesos de toma de decisiones del FPE;

— una evaluación de la eficacia del sistema de gestión de riesgos;

- gestión de conflictos de intereses;

- una evaluación de las necesidades globales de financiación del FPE, incluida una descripción del plan de recuperación cuando ello sea aplicable;

${ }^{3}$ Artículo 64 del Real Decreto 304/2004, de 20 de febrero, por el que se aprueba el Reglamento de planes y fondos de pensiones, «BOE» núm. 48, de 25 de febrero de 2004. 
- una evaluación de los riesgos para los partícipes y beneficiarios en relación con el pago de sus prestaciones de jubilación y la eficacia de cualquier medida correctora;

- una evaluación cualitativa de riesgos nuevos o emergentes relacionados con cambio climático, medio ambiente, riesgos sociales, etc;

— una evaluación cualitativa de los riesgos operativos;

\section{II.D. Depositaria}

Un último aspecto del sistema de Gobernanza lo encontramos en la regulación que la Directiva hace de la actividad de depositaría, especialmente en materia de gestión de posibles conflictos de intereses y de la externalización de funciones que podrá realizarse, con autorización previa del supervisor, y bajo determinadas condiciones. La norma permite a los FPE registrados o autorizados en su territorio que encomienden cualesquiera actividades, incluidas funciones clave y la gestión de las inversiones, a otras entidades. A priori, no parece muy acertado que se lleven a cabo estas externalizaciones porque se entiende que diluyen la responsabilidad de la entidad contratada por el Fondo de Pensiones, aunque en nuestra normativa interna ya se introdujo esta posibilidad a través del Real Decreto 681/2014, de 1 de agosto, por el que se modifica el Reglamento de planes y fondos de pensiones, aprobado por Real Decreto 304/2004, de 20 de febrero. Es básico tener en cuenta que los FPE, pueden encomendar cualquier actividad, incluidas las funciones clave y la de gestión, en su totalidad o parte, a prestadores de servicios que actúen en su nombre, pero que siguen siendo éstos plenamente responsables del cumplimiento de todas las obligaciones que les atribuye la Ley.

\section{INFORMACIÓN A PARTÍCIPES Y BENEFICIARIOS}

Se recoge la obligación para los FPE de ofrecer información clara y adecuada a los potenciales partícipes, a los partícipes y a los beneficiarios para fundamentar las decisiones que adopten sobre su jubilación y garantizar un elevado nivel de trasparencia a lo largo de las distintas fases de un plan de pensiones (fase de incorporación al plan, fase de contribución/aportación y fase de jubilación). Se debe proporcionar una información sobre los derechos consolidados, sobre el nivel previsto de las prestaciones de jubilación, sobre los riesgos y ga- 
rantías y los costes. Esta información se adecuará a las necesidades del usurario, con un contenido que deberá plasmarse en la declaración de las prestaciones de pensión (D.P.P.).

Este es otro de los aspectos más importante a destacar de la Directiva, la introducción del informe normalizado denominado «Declaración de Prestaciones de Pensión», se trata de un documento con información sobre las previsiones de prestaciones de pensión basadas en la edad de jubilación especificada del participe.

En cuanto a las estas obligaciones de información que la nueva Directiva impone a los potenciales partícipes, a los partícipes y beneficiarios, supone una novedad importante, ya que, trata de armonizar la información que reciben los partícipes y beneficiarios en toda la Comunidad Europea, a través de un informe normalizado, la ya citada Declaración de las prestaciones de pensión e información complementaria (D.P.P.).

La Directiva exige a los FPE que elaboren un documento, al menos una vez al año, conciso con información relevante para cada partícipe, teniendo en cuenta la naturaleza específica de los sistemas nacionales de pensiones y de la legislación nacional en materia social, laboral y tributaria.

Según la Directiva la declaración de las prestaciones de pensión incluirá, como mínimo, la siguiente información relevante para los partícipes:

- los datos personales del partícipe, incluida una indicación clara de la edad legal de jubilación, la edad de jubilación establecida en el plan de jubilación o calculada por el FPE, o la edad de jubilación fijada por el partícipe según proceda;

- el nombre del FPE y su dirección de contacto, así como la identificación del plan de pensiones del partícipe;

- cuando corresponda, la información relativa a garantías totales o parciales previstas en el plan de pensiones y, si procede, dónde puede consultarse información adicional al respecto;

- información sobre las previsiones de prestaciones de pensión basadas en la edad de jubilación especificada en el primer punto, y una limitación de responsabilidad en el sentido de que estas previsiones pueden diferir del valor final de las prestaciones recibidas. Si las previsiones de prestaciones de pensión se basan en estimaciones económicas, dicha información también deberá incluir el mejor de los casos estimados, así como 
una estimación desfavorable, teniendo en cuenta la naturaleza específica del plan de pensiones;

— información sobre los derechos consolidados o el capital acumulado, teniendo en cuenta la naturaleza específica del plan de pensiones;

- información sobre las aportaciones abonadas por la empresa promotora y el partícipe al plan de pensiones durante los últimos doce meses, como mínimo, teniendo en cuenta la naturaleza específica del plan de pensiones;

- un desglose de los costes deducidos por el FPE durante los últimos doce meses, como mínimo;

— información sobre el nivel de financiación del plan de pensiones en su conjunto.

Esta parte de la Directiva, tiene alguna similitud con las orientaciones de la recomendación séptima del Pacto de Toledo (en su formulación del 25 de enero de 2011), en relación con la información que las Entidades Gestoras de la Seguridad Social han de ofrecer a los cotizantes, respecto de sus futuros derechos de pensión de jubilación ordinaria, así como sobre sus derechos futuros en el ámbito de la previsión social complementaria.

El documento recuerda a la iniciativa, no llevada a término, de enviar una simulación de pensión, medida conocida popularmente en otros países, como Suecia, como la "carta naranja», que es el color del sobre con la misiva en el que la Administración informa al futuro jubilado de sus derechos al retirarse del mercado de trabajo. En esta carta se le informaría a cada trabajador, con suficiente antelación el nivel económico de que disfrutará con su jubilación, para que pueda adoptar las decisiones de ahorro que más convengan a sus intereses.

En relación con la información del propio fondo de pensiones para los partícipes, hay que entender que la información a facilitar debe tener un contenido básico para su mejor comprensión.

No se puede dejar de comentar una de las partes sobre las que se debe informar, de conformidad con el artículo 37 de la Directiva, «Información general a nivel de plan de pensiones»; y se trata de la información en caso de que el plan prevea varias opciones con diferentes perfiles de inversión. Nuestra normativa interna contempla esta posibilidad para casos muy tasados y con una difícil implementación, tanto es así, que en la práctica no es un aspecto que se haya desarro- 
llado, y es fácil de entender, ya que estamos ante un instrumento de ahorro colectivo y no parece muy acertado darle este enfoque relacionado con el ciclo de vida en las inversiones, insistimos en que a nivel nacional tienen poco arraigo, por lo que no debería considerarse como información básica.

«En el caso de los planes en los que los partícipes asuman un riesgo de inversión y que prevean varias opciones con diferentes perfiles de inversión, se informará a los partícipes de las condiciones relativas a la gama de opciones de inversión disponibles, y, si procede, la opción de inversión por defecto y la norma del plan de pensiones para asignar a un determinado partícipe a una opción de inversión.» ${ }^{4}$

Es significativo conocer los requisitos que la Directiva tiene en cuenta a efectos de proporcionar información, así pues, ésta deberá exponerse en un lenguaje claro y comprensible, de fácil lectura, evitando los tecnicismos. Cabe destacar que la Directiva también habilita a los partícipes potenciales, que son las personas que tienen derecho a adherirse a un plan de pensiones, a obtener un similar nivel de información a la de los partícipes en activo. En general, los requisitos de transparencia e información aumentan con la norma comunitaria, por lo que nuestra normativa interna deberá adaptarse favorablemente.

\section{SUPERVISIÓN PRUDENCIAL}

La última parte de la Directiva se centra en una supervisión de los FPE enfocada al futuro y basada en el riesgo, por ello, se velará para que todos ellos tengan una adecuada organización administrativa y contable y unos procedimientos de control interno adecuados.

Deberemos proporcionar a la autoridad competente una mayor información, lo que supone un avance en la mejora de la información y de los datos estadísticos que ahora se proporcionan. El artículo 51 de la Directiva puede suponer una mayor transparencia de los Informes Estadísticos elaborados por nuestro órgano supervisor, la Dirección General de Seguros y Fondos de Pensiones (DGSFP). El resto de aspectos que se regulan en la Directiva en relación a la supervisión ya los tenemos contemplados en nuestra normativa interna con características similares a las que se nos solicitan en la transposición.

${ }^{4}$ Artículo 37.2 de la Directiva (UE) 2016/2341 del Parlamento Europeo y del Consejo de 14 de diciembre de 2016 relativa a las actividades y la supervisión de los fondos de pensiones de empleo (FPE). 


\section{CONCLUSIONES}

Concluyendo, en España deberemos adaptar nuestra normativa interna para dar cumplimiento a lo establecido en la Directiva, antes del 13 de enero de 2019. Tal y como hemos visto, ya tenemos una parte del camino andada, por lo que no será una transposición intensa. Cabe destacar, que esta adaptación será voluntaria para los FPE que gestionen planes de pensiones que en su conjunto cuenten con menos de 100 partícipes. Es difícil concretar esta redacción de la Directiva por el modo en que se regulan las relaciones entre los planes y los fondos de pensiones a efectos de cumplimiento de esta norma, ya que, se refiere a fondos que gestionen planes con menos de 100 partícipes en su conjunto. Cabría interpretarse que se refiere a los planes con menos de 100 partícipes, ya que, de referirse a los fondos, serían muy pocos los que no se verían afectados por la nueva norma, aproximadamente el 4,4\% del total de fondos existentes que son unos 313 .

Si habláramos de planes, en nuestro caso, el número total de planes de pensiones de empleo es de 1.244, de los que 449 cuentan con menos de 100 partícipes, que suponen el 36,5\% del total, el resto de planes, supera esta cifra, tal y como podemos ver en el siguiente cuadro.

\begin{tabular}{l|c|c|c|c}
\hline \multicolumn{4}{c}{ DATOS DE INVERCO 31/03/2018 } \\
\hline TOTAL PLANES DE PENSIONES DE EMPLEO & \multicolumn{4}{|c}{1.244} \\
\hline & \multicolumn{2}{c|}{$\begin{array}{c}\text { CON MENOS DE 100 } \\
\text { Partícipes }\end{array}$} & \multicolumn{2}{c}{$\begin{array}{c}\text { CON MÁS DE 100 } \\
\text { Partícipes }\end{array}$} \\
\hline Total de Planes de Empleo & 449 & $36,5 \%$ & 795 & $63,5 \%$ \\
\hline Total Partícipes & \multicolumn{3}{|c|}{20.727} & \multicolumn{2}{c|}{1.978 .189} \\
\hline
\end{tabular}

Fuente: ${ }^{5}$

En este sentido, no se entiende que el número de partícipes sea el criterio más acertado para excluir a los planes de pensiones de la aplicación de la norma comunitaria, sería mucho más acertado utilizar un criterio que tuviera en cuenta el valor medio de los derechos consolidados de los partícipes, esto es, tener en cuenta el patrimonio medio para decidir si deben aplicar o no la Directiva. Nuestro sistema de planes y fondos de pensiones, cuenta con algunos planes de pen-

${ }^{5}$ Asociación de Instituciones de Inversión Colectiva y Fondos de Pensiones (INVERCO) datos a 31/03/2017.

(C) UNED. Revista de Derecho UNED, núm. 22, 2018 
siones, bien es cierto que no es lo habitual, que tienen menos de 100 partícipes pero que el patrimonio medio de estos partícipes está alrededor de los 110.000 euros, y sería recomendable que a este tipo de planes se les exigiera aplicar la Directiva y cumplir con el sistema de gobernanza propuesto.

En conclusión, es importante que en la transposición de la Directiva se tenga presente la normativa nacional, y en especial, la articulación de la previsión social complementaria en España, donde la gestión de activos se realiza por entidades gestoras y profesionales independientes y donde las comisiones de control están configuradas como órganos de gobierno de los planes y fondos de pensiones formadas por representantes de los trabajadores y de las empresas.

Nuestro modelo debe ser respetado y la transposición no puede reducir derechos y garantías de un sistema que está perfectamente adaptado a la naturaleza de la previsión social complementaria y al tamaño y patrimonio que los planes de pensiones suponen en nuestro modelo global de protección social pública y privada.

El desarrollo de la previsión social en España a través de la negociación colectiva ha permitido una participación de la representación de los partícipes y el promotor en la Comisión de Control. Este modelo se aleja del origen y configuración llevada a cabo en otros países como pudiera ser el modelo anglosajón con los «trustee». En estos órganos, los miembros asumen mayores responsabilidades a la gestión de estos patrimonios mediante la prestación de servicios remunerados y que deberían ser el objetivo principal de esta directiva.

Es positivo apoyar el desarrollo de estos procedimientos prudenciales, y deben compartirse los objetivos de la Directiva, entre ellos la formación permanente de los miembros de las comisiones de control y la mejora de la información a partícipes y beneficiarios. Pero la Administración deberá tener en cuenta que la transposición de la directiva no puede implicar cargas relevantes adicionales a los representantes de los partícipes y beneficiaros, sino una adaptación de los controles de riesgos existentes y una mejora del contenido de las revisiones financieros actuariales, sólo así, podremos transmitir la idea de que la simplificación puede suponer un mayor desarrollo de estos procedimientos. 